10

\title{
Chromatin condensation, fragmentation of DNA and differences in the epigenetic signature of infertile men
}

\author{
Monica Muratori, Research Associate ${ }^{\mathrm{a}, \text { *, }}$ \\ Christian De Geyter, Professor ${ }^{\text {b }}$ \\ ${ }^{a}$ Department of Experimental, Clinical and Biomedical Sciences, Unit of Sexual Medicine and Andrology, \\ Center of Excellence DeNothe, University of Florence, Viale Pieraccini, 6, I-50139, Firenze, Italy \\ ${ }^{\mathrm{b}}$ Reproductive Medicine and Gynecological Endocrinology (RME), University Hospital, University of Basel, \\ Vogesenstrasse 134, CH-4031, Basel, Switzerland
}

\section{A R T I C L E I N F O}

\section{Article history:}

Available online 3 November 2018

\section{Keywords:}

sperm DNA fragmentation

chromatin condensation

epigenome

endocrine disruptors

male infertility
Epidemiological studies report an increase of pathologies of male reproductive tracts and suggest a link between this trend and the increased exposure of men to endocrine disruptors (EDs). The mechanisms by which EDs impact male fertility are far to be elucidated although DNA, chromatin and epigenome of spermatozoa appear to be relevant targets for these molecules. Indeed, many studies report associations between increased levels of sperm DNA fragmentation (sDF) or aberrant chromatin condensation or epigenetic modifications and poor semen quality and/or infertile phenotype. In this scenario, therapies able to reduce sperm damage to DNA, chromatin and epigenome are sought. Currently, antioxidants and FSH administration is proposed for treating high levels of sDF, but whether or not such therapies are really effective is still debated. Further studies are necessary to understand the link between endocrine disruptor exposure and damage to sperm function and/or structure and thus to define effective therapeutic strategies.

(C) 2018 Published by Elsevier Ltd.

\footnotetext{
* Corresponding author.

E-mail addresses: monica.muratori@unifi.it (M. Muratori), Christian.DeGeyter@usb.ch (C. De Geyter).
} 


\section{Introduction}

Couple infertility has become a top priority for the public health, as one in every four couples experiences problems to conceive [1]. The causes of couple infertility are attributed in equal parts to the female and male partners, and male infertility affects about $7 \%$ of men, including $6-27 \%$ of cases with unexplained male factor infertility [2]. Epidemiological trends in the last decades indicate an increase of the pathologies of male reproductive tracts, including impaired spermatogenesis, testicular cancer, cryptorchidism and hypospadias [3,4]. These trends reflect changes in lifestyle factors such as intense use of cell phone, smoking habit, obesity and physical inactivity [5]. However, a major risk for male reproductive health comes from the spread contamination of water, soil, air, food, beverages and household exposing humans to many environmental toxicants, including endocrine disruptors (EDs). EDs mimic or block or interfere with the synthesis, secretion, transport, metabolism or degradation of the endogenous hormones [6] and consist in a wide range of substances, both natural and synthetic, including air pollutants, pharmaceuticals, dioxin and dioxin-like compounds, polychlorinated biphenyls, dichlorodiphenyltrichloroethane (DDT) and other pesticides, and plasticizers such as bisphenol A.

The damaging effects of environmental contaminants on male infertility have been indicated by several studies conducted on animals or humans. Investigations carried out on animals reported decreases in daily production and DNA integrity of spermatozoa after exposure to car exhaust [7] or air pollution [8]. In human, it has been reported that blood concentrations of polychlorinated biphenyls and related metabolites negatively correlated with sperm count and motility [9]. Similarly, Bloom and coworkers found that urinary concentrations of some monoester metabolites of phthalate diesters are associated with lower sperm count and abnormal sperm morphology [10], whereas exposure to high air pollution increased sperm DNA damage in policemen working in the center of Praga [11]. Poor semen quality may occur also in consequence of exposure to EDs during foetal development, when a decrease of androgen action might damage Sertoli and Leydig cell function, in turn responsible for the onset of clinical reproductive disorders in the adult life [4].

The mechanisms through which pollution impacts male fertility are far to be fully elucidated, but DNA, chromatin and epigenome of spermatozoa appear to be major targets for the action of environmental toxicants, including EDs.

\section{Sperm DNA fragmentation}

Sperm DNA fragmentation (sDF) consists of both single and double stranded DNA breaks in the sperm nuclei. High levels of sDF can be present in semen with normal motility, morphology and count, indicating that this type of sperm damage is independent from conventional semen parameters [12] and may underlie cases of idiopathic male infertility. In animals, the negative effect of high levels of sDF on reproduction and health of the offspring is well known [13]. In humans, Evenson and coworkers first reported both the delayed time to pregnancy in infertile couples or complete infertility, when the male partner shows increased levels of sDF [14]. Subsequent reports confirmed this result using different techniques to reveal sDF $[12,15]$ and in couples treated by treated by intrauterine insemination $[16,17]$. Conversely, data on the relationship between increased levels of sDF and early reproductive outcomes after In vitro Fertilization (IVF) and Intracytoplasmic Sperm Injection (ICSI) are conflicting [18]. However, two recent meta-analyses reported that high levels of sDF reduce the probability of clinical pregnancy after IVF and ICSI [19,20]. In addition, it is well established that the risk of miscarriage increases with sDF in couples who conceive spontaneously or after Assisted Reproduction Technology (ART) [21].

\section{Origin of $s D F$}

The pathways leading to increased levels of sDF are numerous and involve diseases, lifestyle factors, drugs, aging and leukocytospermia [22]. In addition, many epidemiological and occupational studies identified several environmental chemicals able to induce sDF. Many of these reproductive toxicants 
are EDs and include pesticides such as organophosphates, phthalates, polychlorinated biphenyls, and metals such as lead [23].

All the pathways leading to sperm DNA damage converge on one of three main cell mechanisms i.e. abortive apoptosis, defect in sperm chromatin maturation and oxidative stress. Abortive apoptosis originates in testis, proceeds up to germ cell DNA cleavage but fails to delete committed cells to death and thus spermatozoa with apoptotic traits can be found in the ejaculate [24]. Whether or not an apoptotic process may occur also after spermiation being responsible of extra-testicular generation of $\mathrm{sDF}$ is a controversial issue, as the strict compaction of the sperm nuclei might prevent the action of apoptotic nucleases [25]. Defects in sperm chromatin maturation refer to a failure of repairing those DNA nicks that are produced to promote histone to protamine transition during sperm nuclei remodeling [26] in spermatids. Finally, the third mechanism originating sDF is sperm oxidative stress, occurring when Reactive Oxygen Species (ROS) production overwhelms the antioxidant defenses [22]. Oxidative stress seems to be the main mechanism acting during sperm transit in the male genital tract as ejaculated spermatozoa exhibit higher levels of sDF than testicular ones [27].

A recent study investigated the relative contribution of these three mechanisms to the origin of sDF by detecting the co-presence, in the single spermatozoon, of DNA breakage and characteristic traits of apoptosis, chromatin maturation failure and oxidative damage [28]. In native semen samples, sDF highly overlapped with apoptosis and chromatin immaturity, indicating that both apoptotic cleavage and failure in chromatin maturation play a role in causing sperm DNA breaks. Conversely, overlapping between SDF and oxidative damage to nucleus or to membrane was present only in few spermatozoa, suggesting that DNA breakage occurs independently from any oxidative attack [28]. This scenario changed considerably when the co-presence of SDF and oxidative damage was studied in the viable fraction of native semen samples. Indeed, viable spermatozoa with sDF exhibited oxidative DNA/ membrane damage, suggesting that an oxidative assault can be responsible for DNA breakage in the viable fraction of the ejaculate [28]. These results must be interpreted keeping in mind that in native semen samples most of the ejaculated DNA fragmented spermatozoa are dead [29]. These spermatozoa are likely cells beginning DNA breakage because of testicular apoptosis, and arriving moribund or dead in the male genital tract where thus cannot be injured by a possible oxidative environment [30] (Fig. 1). Conversely, an oxidative milieu in male genital tract may attack viable spermatozoa [30] and induce DNA breakage (Fig. 1).

\section{Sperm chromatin condensation}

In male germ cells, chromatin architecture undergoes a dramatic remodeling during the maturation of spermatids to spermatozoa, when histones are nearly completely substituted by protamines. This process leads to a unique nuclear architecture where chromatin is organized in very stable toroidal structures and sperm DNA is six times more condensed than in somatic nuclei [31]. To accomplish this, histones undergone hyper-acetylation in order to reduce their DNA binding affinity and thus promoting their displacement [32]. After hyper-acetylation, histones are first replaced by transition proteins and then by protamines [33]. Only a small amount of DNA (about 15\% in human spermatozoa) remains associated to histones maintaining the nucleosomal structure [34]. The highly compacted sperm chromatin structure guarantees that mature spermatozoa acquire a hydrodynamic nuclear shape and that paternal genome will be delivery safely to the oocyte. However, chromatin remodeling has also a pivotal role in the epigenetic reprogramming of paternal DNA after fertilization [35]. Protamines bind DNA very tightly and disulphide bonds between cysteine residues of adjacent protamines further condense sperm chromatin. In humans, there are two types of protamines: protamine 1 (P1) and protamine 2 (P2) and defects in their expression and processing may alter sperm chromatin condensation and affect male fertility. Similarly, abnormal P1:P2 ratios (the normal ratio is $0.8-1.2$ ) has been associated to poor semen quality, increased SDF, reduced sperm penetration ability and male infertility [33]. Impairment of a proper histone to protamine replacement, decreasing sperm chromatin condensation, is involved in male infertility as well [33]. Indeed, reduction or premature histone hyperacetylation provokes abnormal spermatogenesis [36] and an excessive histone retention is frequently observed in infertile men [37]. In couples treated by ART, the amount of spermatozoa with proper chromatin condensation is positively associated to fertilization rate [38,39], embryo quality [40] and 
TESTIS SPERMIATION EJACULATION
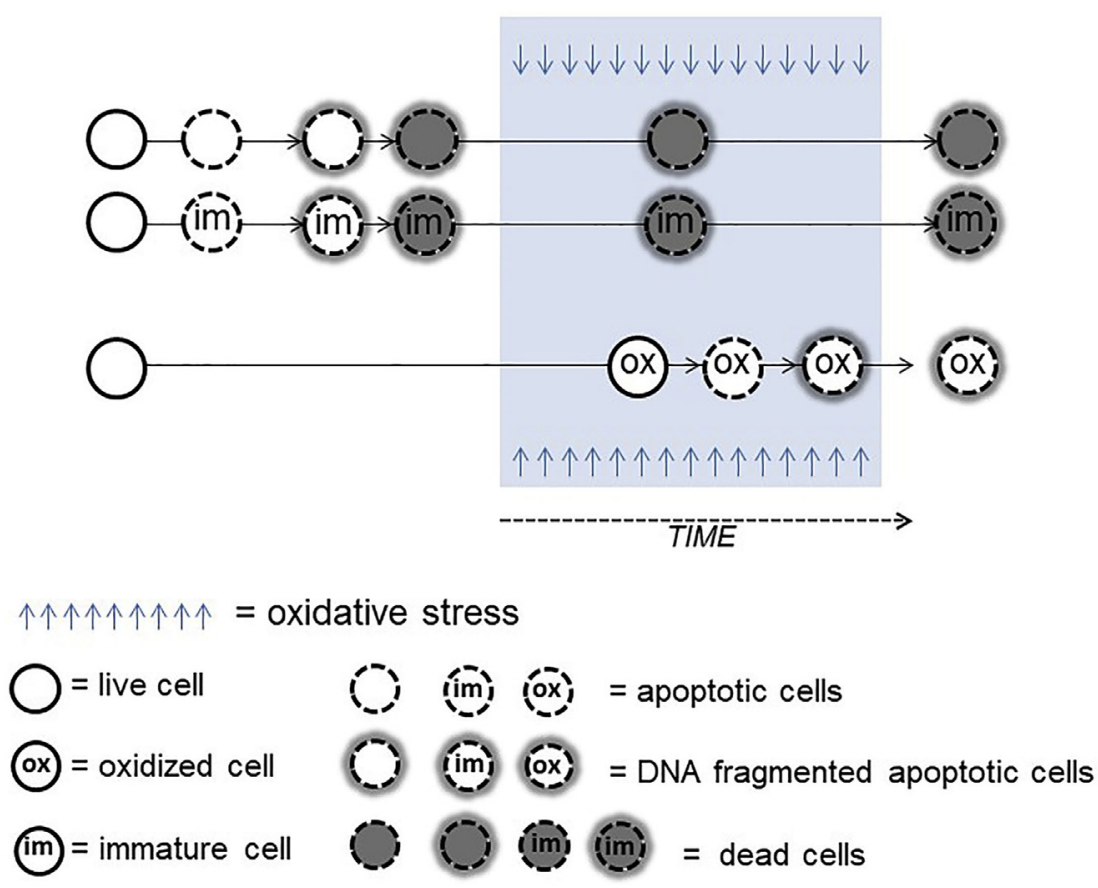

Fig. 1. Origin of sperm DNA fragmentation. Several stimuli, including impairment of chromatin maturation, can trigger apoptosis in germ cells and thus DNA fragmentation and death (rows 1-2). These cells go through an eventual oxidative milieu in male genital tracts without injures. Conversely, oxidative species attack live cells, leading them to apoptosis and DNA fragmentation (row 3 ).

clinical pregnancy [41]. In addition, sperm nuclear decondensation is higher in the male partners of couples with recurrent pregnancy loss than in fertile subjects [42].

EDs may interfere with maturation of sperm chromatin, as reported by studies both in animals and humans. Chronical exposure to vinclozolin in rats disrupted both the sperm nuclear morphology and the chromatin texture, which are related to the status of nuclear condensation [43], and similar results were reported after treating rats for 3-6 weeks with bisphenol A [44]. In human, environmental exposure to DDT provoked an improper sperm chromatin condensation in $46.6 \%$ of exposed subjects and the blood concentrations of dichlorodiphenyldichloroethylene (DDE, the major metabolite of DTT) correlated with the amount of the most severe sperm chromatin decondensation [45]. Finally, some studies reported that lead competes or replaces the $\mathrm{Zn}$ in protamines, changing protein conformation and thus provoking poorly condensed sperm nuclei $[46,47]$.

\section{Epigenetic changes}

There is now accumulating evidence that epigenetics could be a major mechanism to mediate the environmental impact on male infertility and on the health of the offspring. The most impactful action of environmental factors on germ line occurs in developmental windows of sensitivity, namely during the prenatal differentiation of primordial germ cells and during the differentiation of germ cells into spermatogonia [48]. However, as spermatogenesis is a life-long ongoing process, a third window of sensitivity occurs also in the adult male $[49,50]$. Aberrations in the sperm epigenome may provoke adverse effects on male fertility and on embryogenesis [51] and can be perpetuated across generations 
(transgenerational inheritance). The main epigenetic mechanisms include DNA methylation, histone modifications and post-transcriptional regulation by micro RNAs.

\section{DNA methylation in spermatozoa}

DNA methylation mainly occurs on cytosine residues at cytosine-phosphate-guanine (CpG) dinucleotides and is a powerful regulator of gene transcription. The methylation status of spermatozoa is the results of the incomplete erasure of DNA methylation in the primordial germ cells in early embryo development, and the de novo DNA methylation during all stages of sperm maturation [52]. It has been extensively reported that anomalies of DNA methylation in the global sperm genome or in specific gene loci is associate with poor semen quality [53], unexplained male infertility [54] or poor reproductive outcome [55,56]. In addition, incorrect methylation in imprinted and non-imprinted genes is associated with male infertility [57,58]. Environmental factors, including lifestyle and exposure to toxicants, can induce changes in sperm DNA methylation. For instance, both physical exercise and obesity are able to alter methylation of genes involved in nervous system development [52]. In animal, exposure during foetal gonadal sex determination to several plastics derived EDs associated to differential methylation at 197 promoters [59]. Similarly, transient exposure in utero to fungicide vinclozolin provoked transgenerational adult onset disease that was correlated to changes of DNA methylation pattern in the sperm epigenome [60]. Finally, administration of methoxychlor to adult male mice induced defects of methylation of several maternally and paternally imprinted genes in sperm DNA [61].

\section{Histone modification}

Histone modifications represent another basic mechanism of epigenetic regulation. They consist of histone variants as well as tail modifications to lysine and serine residues of histone proteins (posttranslational modifications, PTMs). Histone modifications regulate gene transcription by altering chromatin structure, in turn modulating gene expression $[62,63]$.

As mentioned, spermatids undergo intense chromatin remodeling during the histone to protamine transition, affecting about $85 \%$ of all DNA. Although excessive histone retention is considered a sign of sperm chromatin immaturity, emerging evidence supports the fact that position of the remaining histones in 15\% of DNA is not randomly distributed and possibly plays an important role in sperm function [52,64]. In particular, retained histones are located in telomeric and pericentromeric chromosome sites where they play a structural role [34,65], although at least a portion of histones is retained at promotor regions and near genes involved in embryo development $[66,67]$.

In male germ cells, the expression of a large number of non-canonical histone variants may mediate an epigenetic influence by environmental factors. Histone variants differ from canonical histones by small amino acid sequences, are differentially expressed during germ cells development and play both structural and gene regulatory functions [68]. Some of these variants (for instance H2AL1 and H2AL2) seem to play a role only during spermatogenesis, whereas others might represent epigenetic marks transmitted to the next generation [69].

Histone PTMs consist of methylation, acetylation, phosphorylation and other modifications of the $\mathrm{N}$-terminal domain of these proteins. Their occurrence in the octamer core of the nucleosomes alters the electrostatic charge of chromatin, thus influencing the structural organization of chromatin and DNA accessibility to transcription factors or adaptor proteins [70,71]. Hence, histone PTMs and the network of the enzymes adding and removing tail modifications appear to be important players in the epigenetic control of germ cell development [72]. Defects in the histone PTMs are associated to male infertility. It has been reported that alterations in histone acetylation and/or methylation result in an improper sperm chromatin condensation [73] and a reduced expression of Brdt [74], a gene playing an important role for establishing germ cell differentiation. Similarly, inhibition or loss of activity of the enzymes catalyzing PTMs associates to a decrease of number of spermatids, germ cell apoptosis and severe male infertility [75].

Environmental toxicants could induce alterations in the sperm epigenome by acting on histone positioning and modification, as already reported for diet induced factors [76]. In addition, as protamines erase epigenetic marks due to the removed histones, also protamination participates to sperm 
epigenome and may represent another mechanism mediating environmental influences [52], albeit vastly unknown.

\section{Micro RNAs}

Micro RNAs (miRNAs) are the most well-characterized small RNAs (sRNAs), i.e. non coding RNA species regulating gene expression by interfering at translational level and/or degrading their complementary mRNAs [52]. The occurrence of RNA species in mature spermatozoa has been long interpreted as remnants of molecules of immature germ cells. After the pioneering study by Krawetz's group [77], indicating that the male gamete passes paternal transcripts to the oocyte during fertilization, it is now clear that sperm borne miRNAs play an important role in the early embryo development $[78,79]$. In addition, miRNAs are essential molecules for a normal sperm production, as they participate in the control of each step of spermatogenesis [80]. In this scenario, it is not surprising that aberrant expression of miRNAs negatively impact male fertility. Compared to fertile men, alterations in seminal plasma miRNA profiles were found in infertile men with azoo-, astheno- [81] and oligozoospermia [82]. In addition, changes in the expression of specific mRNAs are associated to germ cell arrest and increased rate of testis apoptosis, both leading to male infertility [83].

It has been extensively reported that miRNA expression and function in somatic cells is deeply affected by environmental contaminants $[84,85]$. Although specific studies on germ cell line have not been published so far, it can be anticipated that environmental exposure to toxicants yields similar effect in miRNA expression during spermatogenesis.

\section{Conclusive remarks}

Human are exposed to a wide number of environmental pollutants, including the reproductive toxicants EDs. Many studies indicate that EDs induce sDF, improper chromatin condensation and epigenetic modifications. All these effects impact sperm functions and male fertility status with relevant consequences for reproduction. Importantly, a crosstalk between the pathways impairing DNA, chromatin integrity and epigenome of spermatozoa can occur during spermatogenesis. It is well known the link between poor chromatin condensation and the higher susceptibility to ROS attack, a cause of sDF [22]. On the other hand, a derailment in chromatin maturation can trigger apoptosis in the testis [28] and, vice versa, maturation fails in germ cells committed to apoptosis [86]. In addition, any impairment in histone to protamine transition, and thus chromatin condensation, may yield epigenetic perturbations, as protamine positioning may represent an epigenetic mark as well. Further, it is known from animal models that several EDs stimulate testicular ROS generation [87,88], which can induce both DNA breaks and changes in the epigenome of spermatozoa [89]. Finally, aberrant expression of small non coding RNA, including miRNAs [83], or of histone PTMs [75], are associated with impaired germ cell function such as increased apoptosis, the main testicular mechanism originating sDF.

Strategies to prevent or treat sDF, aberrant chromatin condensation and epigenetic modifications are sought, however we are still far from this goal. Indeed, the molecular mechanisms by which EDs damage sperm structures and functions are not fully elucidated. Currently, therapies for high levels of sDF are available but their effectiveness is debated. Indeed, although the use of antioxidants has shown some positive effects, clear conclusions cannot be drawn because of the several limitations of the studies on the outcomes of these therapies [90]. Similarly, the effectiveness of FSH administration in targeting testicular apoptosis is debated, due to the presence of non-responding subjects [91,92].

In the ART setting, the procedures to prepare semen should yield sperm populations with higher nuclear maturity and DNA integrity, other than motility. Indeed, many studies reported a decrease in sperm nuclear immaturity [93] or sDF [94] after selection with swim up or density gradient centrifugations, the two popular techniques to select spermatozoa for ART. In case of sDF, however, some groups observed that these procedures can even induce DNA damage [95-97] and, most importantly, subjects increasing the damage during selection experience a $50 \%$ lower probability to achieve pregnancy [97].

In recent years, advanced procedures to prepare spermatozoa with increased maturity and DNA integrity have been proposed. These procedures select spermatozoa based on surface charge or 
external exposure of phosphatidylserine or binding sites for hyaluronic acid [98]. Another selection procedure takes advantage from the fact that mature sperm nuclei have high intrinsic birefringence and can be recognizable with suitable microscopes [99]. Finally, ultra-high magnification microscopy has been proposed in ICSI to select spermatozoa devoid of subtle defects in cell structures [100]. However, despite individual small studies reporting clinical benefits with these procedures in terms of ART outcomes, a clear conclusion cannot be drawn. Indeed, Cochrane reviews [98,100] reported that there is insufficient evidence or that studies of suitable quality are still lacking to demonstrate that any of these procedures can improve the clinical results.

In conclusion, the wide diffusion of environmental toxicants threatens male reproductive health by deeply affecting DNA integrity, chromatin density and epigenetic signature of human spermatozoa. To now, no fully effective therapy is available to treat these types of sperm damage.

\section{Summary}

Widespread environmental contamination threats male reproductive health. In particular, exposure to EDs may be responsible for the decline in sperm count and the increase of testicular cancer, cryptorchidism and hypospadias. The mechanisms by which EDs impact male fertility are not fully clarified but DNA, chromatin and epigenome of spermatozoa appear to be relevant targets for these molecules. Indeed, many studies report that the exposure to EDs induces sDF and chromatin decondensation and, on the other hand, it is well known that high levels of sDF reduce the probability to achieve pregnancy and increase the risk of miscarriage after natural or assisted conception. Similarly, an impaired sperm chromatin condensation negatively affects reproduction as well. Further, emerging evidence indicates that exposure to EDs impacts on male fertility through changes in DNA methlylation, histone modifications and, likely, miRNA profiles of spermatozoa. However, the pathways by which EDs damage these sperm traits are not fully understood and therapies for these types of damage are far to be developed. Although, antioxidants and FSH have been proposed to target testis apoptosis and oxidative stress, two main mechanisms originating sDF, the effectiveness of these therapies is still debated. In addition, in the ART setting, it is not sure that the popular procedures to prepare semen ameliorate sperm DNA integrity, and the advantage of using sophisticated selection techniques to improve reproductive outcomes remains controversial. Hence, further efforts are necessary to address possible strategies to prevent or treat sperm damage due to environmental contaminant exposure.

\section{Research agenda}

- Exposure to environmental toxicant is a threat for reproductive male health

- Further studies are necessary to fully understand the mechanisms by which endocrine disruptors impact sperm structure and function.

- Effective strategies to treat or prevent DNA fragmentation, chromatin decondensation and epigenetic modifications of spermatozoa are sough, but we are still far from this goal.

\section{Acknowledgments}

Fig. 1 has been adapted from the original article "[Investigation on the Origin of Sperm DNA Fragmentation: Role of Apoptosis, Immaturity and Oxidative Stress]" by [Muratori M, Tamburrino L, Marchiani S, Cambi M, Olivito B, Azzari C, Forti G, Baldi E]. Molecular Medicine 2015; 21:109-22 (https://doi.org/10.2119/molmed.2014.00158; http://static.smallworldlabs.com/molmedcommunity/ content/pdfstore/14_158_Muratori.pdf). The original article is an open access article distributed under the terms of the Creative Commons Attribution License (http://creativecommons.org/licenses/ by/2.0), which permits unrestricted use, distribution, and reproduction in any medium, provided the original work is properly cited. 


\section{References}

[1] World Health Organization. Infertility is a global public health issue. 2017. http://www.who.int/reproductivehealth/ topics/infertility/perspective/en/.

[2] Hamada A, Esteves SC, Agarwal A. Unexplained male infertility: potential causes and management. Hum Androl 2011; $1: 2-16$.

*[3] Levine H, Jørgensen N, Martino-Andrade A, et al. Temporal trends in sperm count: a systematic review and metaregression analysis. Hum Reprod Update 2017;23:646-59.

*[4] Skakkebaek NE, Rajpert-De Meyts E, Buck Louis GM, et al. Male reproductive disorders and fertility trends: influences of environment and genetic susceptibility. Physiol Rev 2016;96:55-97.

*[5] Barazani Y, Katz BF, Nagler HM, et al. Lifestyle, environment, and male reproductive health. Urol Clin North Am 2014; 41:55-66.

[6] Bonde JP, Flachs EM, Rimborg S, et al. The epidemiologic evidence linking prenatal and postnatal exposure to endocrine disrupting chemicals with male reproductive disorders: a systematic review and meta-analysis. Hum Reprod Update 2016;23:104-25.

[7] Yoshida S, Sagai M, Oshio S, et al. Exposure to diesel exhaust affects the male reproductive system of mice. Int J Androl 1999;22:307-15.

[8] Yauk C, Polyzos A, Rowan-Carroll A, et al. Germline mutations, DNA damage, and global hypermethylation in mice exposed to particulate air pollution in an urban/industrial location. Proc Natl Acad Sci U S A 2008;105:605-10.

[9] Dallinga JW, Moonen EJ, Dumoulin JC, et al. Decreased human semen quality and organochlorine compounds in blood. Hum Reprod 2002;17:1973-9.

[10] Bloom MS, Whitcomb BW, Chen Z, et al. Associations between urinary phthalate concentrations and semen quality parameters in a general population. Hum Reprod 2015;30:2645-57.

[11] Rubes J, Selevan SG, Evenson DP, et al. Episodic air pollution is associated with increased DNA fragmentation in human sperm without other changes in semen quality. Hum Reprod 2005;20:2776-83.

[12] Muratori M, Marchiani S, Tamburrino L, et al. DNA fragmentation in brighter sperm predicts male fertility independently from age and semen parameters. Fertil Steril 2015;104:582-90. e4.

[13] Ramos-Ibeas P, Calle A, Fernández-González R, et al. Intracytoplasmic sperm injection using DNA-fragmented sperm in mice negatively affects embryo-derived embryonic stem cells, reduces the fertility of male offspring and induces heritable changes in epialleles. PloS One 2014;9:e95625.

[14] Evenson DP, Jost LK, Marshall D, et al. Utility of the sperm chromatin structure assay as a diagnostic and prognostic tool in the human fertility clinic. Hum Reprod 1999;14:1039-49.

[15] Simon L, Brunborg G, Stevenson M, et al. Clinical significance of sperm DNA damage in assisted reproduction outcome. Hum Reprod 2010;25:1594-608.

[16] Bungum M, Humaidan P, Axmon A, et al. Sperm DNA integrity assessment in prediction of assisted reproduction technology outcome. Hum Reprod 2007;22:174-9.

[17] Boe-Hansen GB, Fedder J, Ersbøll AK, et al. The sperm chromatin structure assay as a diagnostic tool in the human fertility clinic. Hum Reprod 2006;21:1576-82.

[18] Tamburrino L, Marchiani S, Montoya M, et al. Mechanisms and clinical correlates of sperm DNA damage. Asian J Androl $2012 ; 14: 24-31$.

*[19] Simon L, Zini A, Dyachenko A, et al. A systematic review and meta-analysis to determine the effect of sperm DNA damage on in vitro fertilization and intracytoplasmic sperm injection outcome. Asian J Androl 2017;19:80-90.

[20] Cissen M, Wely MV, Scholten I, et al. Measuring sperm DNA fragmentation and clinical outcomes of medically assisted reproduction: a systematic review and meta-analysis. PloS One 2016;11, e0165125.

[21] Robinson L, Gallos ID, Conner SJ, et al. The effect of sperm DNA fragmentation on miscarriage rates: a systematic review and meta-analysis. Hum Reprod 2012;27:2908-17.

[22] Aitken RJ, Bronson R, Smith TB, et al. The source and significance of DNA damage in human spermatozoa; a commentary on diagnostic strategies and straw man fallacies. Mol Hum Reprod 2013;19:475-85.

[23] Barratt CL, Aitken RJ, Björndahl L, et al. Sperm DNA: organization, protection and vulnerability: from basic science to clinical applications-a position report. Hum Reprod 2010;25:824-38.

[24] Sakkas D, Mariethoz E, St John JC. Abnormal sperm parameters in humans are indicative of an abortive apoptotic mechanism linked to the Fas-mediated pathway. Exp Cell Res 1999;251:50-5.

[25] Koppers AJ, De Iuliis GN, Finnie JM, et al. Significance of mitochondrial reactive oxygen species in the generation of oxidative stress in spermatozoa. J Clin Endocrinol Metabol 2008;93:3199-207.

[26] Marcon L, Boissonneault G. Transient DNA strand breaks during mouse and human spermiogenesis new insights in stage specificity and link to chromatin remodeling. Biol Reprod 2004;70:910-8.

[27] Esteves SC, Roque M, Garrido N. Use of testicular sperm for intracytoplasmic sperm injection in men with high sperm DNA fragmentation: a SWOT analysis. Asian J Androl 2018;20:1-8.

*[28] Muratori M, Tamburrino L, Marchiani S, et al. Investigation on the origin of sperm DNA fragmentation: role of apoptosis, immaturity and oxidative stress. Mol Med 2015;21:109-22.

[29] Mitchell LA, De Iuliis GN, Aitken RJ. The TUNEL assay consistently underestimates DNA damage in human spermatozoa and is influenced by DNA compaction and cell vitality: development of an improved methodology. Int J Androl $2011 ; 34: 2-13$.

[30] Aitken RJ, Whiting S, De Iuliis GN, et al. Electrophilic aldehydes generated by sperm metabolism activate mitochondrial reactive oxygen species generation and apoptosis by targeting succinate dehydrogenase. J Biol Chem 2012; 287:33048-60.

[31] Balhorn R, Brewer L, Corzett M. DNA condensation by protamine and arginine-rich peptides: analysis of toroid stability using single DNA molecules. Mol Reprod Dev 2000;56:230-4. 
[32] Hong L, Schroth GP, Matthews HR, et al. Studies of the DNA binding properties of histone H4 amino terminus. Thermal denaturation studies reveal that acetylation markedly reduces the binding constant of the H4 "tail" to DNA. J Biol Chem 1993;268:305-14.

[33] Francis S, Yelumalai S, Jones C, et al. Aberrant protamine content in sperm and consequential implications for infertility treatment. Hum Fertil (Camb) 2014;17:80-9.

[34] Gatewood J, Cook G, Balhorn R, et al. Sequencespecific packaging of DNA in human sperm chromatin. Science 1987; 236:962-4.

*[35] Oliva R. Protamines and male infertility. Hum Reprod Update 2006;12:417-35.

[36] Sonnack V, Failing K, Bergmann M, et al. Expression of hyperacetylated histone H4 during normal and impaired human spermatogenesis. Andrologia 2002;34:384-90.

[37] Zhang X, San Gabriel M, Zini A. Sperm nuclear histone to protamine ratio in fertile and infertile men: evidence of heterogeneous subpopulations of spermatozoa in the ejaculate. J Androl 2006;27:414-20.

[38] Nasr-Esfahani MH, Razavi S, Mardani M. Relation between different human sperm nuclear maturity tests and in vitro fertilization. J Assist Reprod Genet 2001;18:219-25.

[39] Tavalaee M, Razavi S, Nasr-Esfahani MH. Influence of sperm chromatin anomalies on assisted reproductive technology outcome. Fertil Steril 2009;91:1119-26.

[40] Marchiani S, Tamburrino L, Benini F, et al. Chromatin protamination and catsper expression in spermatozoa predict clinical outcomes after assisted reproduction programs. Sci Rep 2017;7:15122.

[41] Irez T, Sahmay S, Ocal P, et al. Investigation of the association between the outcomes of sperm chromatin condensation and decondensation tests, and assisted reproduction techniques. Andrologia 2015;47:438-47.

[42] idi-Jrah I, Hajlaoui A, Mougou-Zerelli S, et al. Relationship between sperm aneuploidy, sperm DNA integrity, chromatin packaging, traditional semen parameters, and recurrent pregnancy loss. Fertil Steril 2016;105:58-64.

[43] Auger J, Lesaffre C, Bazire A, et al. High-resolution image cytometry of rat sperm nuclear shape, size and chromatin status. Experimental validation with the reproductive toxicant vinclozolin. Reprod Toxicol 2004;18:775-83.

[44] Othman AI, Edrees GM, El-Missiry MA, et al. Melatonin controlled apoptosis and protected the testes and sperm quality against bisphenol A-induced oxidative toxicity. Toxicol Ind Health 2016;32:1537-49.

[45] De Jager C, Farias P, Barraza-Villarreal A, et al. Reduced seminal parameters associated with environmental DDT exposure and p,p'-DDE concentrations in men in Chiapas, Mexico: a cross-sectional study. J Androl 2006;27:16-27.

[46] Apostoli P, Telišman S, Sager PR. Reproductive and developmental toxicity of metals. In: Nordberg GF, Fowler BA, Nordberg M, et al., editors. Handbook on the toxicology of metals. Amsterdam: Academic Press Elsevier; 2007. p. 213-49.

[47] Hernández-Ochoa I, Sánchez-Gutiérrez M, Solís-Heredia MJ, et al. Spermatozoa nucleus takes up lead during the epididymal maturation altering chromatin condensation. Reprod Toxicol 2006;21:171-8.

[48] Ly L, Chan D, Trasler JM. Developmental windows of susceptibility for epigenetic inheritance through the male germline. Semin Cell Dev Biol 2015;43:96-105.

[49] Rando OJ. Daddy issues: paternal effects on phenotype. Cell 2012;151:702-8.

[50] Rando OJ. Intergenerational transfer of epigenetic information in sperm. Cold Spring Harbor Perspect Med 2016;6: a022988.

[51] Hammoud SS, Nix DA, Zhang H, et al. Distinctive chromatin in human sperm packages genes for embryo development. Nature 2009;460:473-8.

*[52] Donkin I, Barrès R. Sperm epigenetics and influence of environmental factors. Molecular Metabolism 2018. pii: S22128778(18)30104-30112.

[53] Kobayashi H, Sato A, Otsu E, et al. Aberrant DNA methylation of imprinted loci in sperm from oligospermic patients. Hum Mol Genet 2007;16:2542-51.

[54] Urdinguio RG, Bayón GF, Dmitrijeva M, et al. Aberrant DNA methylation patterns of spermatozoa in men with unexplained infertility. Hum Reprod 2015;30:1014-28.

[55] Laurentino S, Borgmann J, Gromoll J. On the origin of sperm epigenetic heterogeneity. Reproduction 2016;151:R71-8.

[56] Manipalviratn S, DeCherney A, Segars J. Imprinting disorders and assisted reproductive technology. Fertil Steril 2009; 91:305-15.

*[57] Guerrero-Bosagna C, Skinner MK. Environmentally induced epigenetic transgenerational inheritance of male infertility. Curr Opin Genet Dev 2014;26:79-88.

[58] Houshdaran S, Cortessis VK, Siegmund K, et al. Widespread epigenetic abnormalities suggest a broad DNA methylation erasure defect in abnormal human sperm. PloS One 2007;2:e1289.

[59] Manikkam M, Tracey R, Guerrero-Bosagna C, et al. Plastics derived endocrine disruptors (BPA, DEHP and DBP) induce epigenetic transgenerational inheritance of obesity, reproductive disease and sperm epimutations. PloS One 2013;8: e55387.

[60] Guerrero-Bosagna C, Covert TR, Haque MM, et al. Epigenetic transgenerational inheritance of vinclozolin induced mouse adult onset disease and associated sperm epigenome biomarkers. Reprod Toxicol 2012;34:694-707.

[61] Stouder C, Paoloni-Giacobino A. Specific transgenerational imprinting effects of the endocrine disruptor methoxychlor on male gametes. Reproduction 2011;141:207-16.

[62] Nguyen AT, Zhang Y. The diverse functions of Dot1 and H3K79 methylation. Genes Dev 2011;25:1345-58.

[63] Wolffe AP, Hayes JJ. Chromatin disruption and modification. Nucleic Acids Res 1999;27:711-20.

[64] Hammoud SS, Nix DA, Hammoud AO, et al. Genome-wide analysis identifies changes in histone retention and epigenetic modifications at developmental and imprinted gene loci in the sperm of infertile men. Hum Reprod 2011; 26:2558-69.

[65] Zalenskaya IA, Bradbury EM, Zalensky AO. Chromatin structure of telomere domain in human sperm. Biochem Biophys Res Commun 2000;279:213-8.

[66] Arpanahi A, Brinkworth M, Iles D, et al. Endonuclease-sensitive regions of human spermatozoal chromatin are highly enriched in promoter and CTCF binding sequences. Genome Res 2009;19:1338-49. 
[67] Carone BR, Hung JH, Hainer SJ, et al. High-resolution mapping of chromatin packaging in mouse embryonic stem cells and sperm. Dev Cell 2014;30:11-22.

*[68] Meyer RG, Ketchum CC, Meyer-Ficca ML. Heritable sperm chromatin epigenetics: a break to remember. Biol Reprod 2017;97:784-97.

[69] Wu F, Caron C, De Robertis C, et al. Testis-specific histone variants H2AL1/2 rapidly disappear from paternal heterochromatin after fertilization. J Reprod Dev 2008;54:413-7.

[70] Banister CE, Koestler DC, Maccani MA, et al. Infant growth restriction is associated with distinct patterns of DNA methylation in human placentas. Epigenetics 2011;6:920-7.

[71] Sadakierska-Chudy A, Kostrzewa RM, Filip M. A comprehensive view of the epigenetic landscape part I: DNA methylation, passive and active DNA demethylation pathways and histone variants. Neurotox Res 2015;27:84-97.

[72] Deb M, Sengupta D, Kar S, et al. Epigenetic drift towards histone modifications regulates CAV1 gene expression in colon cancer. Gene 2016;581:75-84.

[73] Paradowska AS, Miller D, Spiess AN, et al. Genome wide identification of promoter binding sites for H4K12ac in human sperm and its relevance for early embryonic development. Epigenetics 2012;7:1057-70.

[74] Steilmann C, Cavalcanti MC, Bartkuhn M, et al. The interaction of modified histones with the bromodomain testisspecific (BRDT) gene and its mRNA level in sperm of fertile donors and subfertile men. Reproduction 2010;140: 435-43.

[75] Das L, Parbin S, Pradhan N, et al. Epigenetics of reproductive infertility. Front Biosci (Schol Ed) 2017 Jun 1;9:509-35.

[76] Carone BR, Fauquier L, Habib N, et al. Paternally induced transgenerational environmental reprogramming of metabolic gene expression in mammals. Cell 2010;143:1084-96.

[77] Ostermeier GC, Miller D, Huntriss JD, et al. Reproductive biology: delivering spermatozoan RNA to the oocyte. Nature 2004;429:154.

[78] Gapp K, Jawaid A, Sarkies P, et al. Implication of sperm RNAs in transgenerational inheritance of the effects of early trauma in mice. Nat Neurosci 2014;17:667-9.

[79] Grandjean V, Fourré S, De Abreu DA, et al. RNA-mediated paternal heredity of diet-induced obesity and metabolic disorders. Sci Rep 2015;5:18193.

[80] Saxe JP, Lin H. Small noncoding RNAs in the germline. Cold Spring Harbor Perspect Biol 2011;3:a002717.

[81] Wang C, Yang C, Chen X, et al. Altered profile of seminal plasma microRNAs in the molecular diagnosis of male infertility. Clin Chem 2011;57:1722-31.

[82] Wu W, Hu Z, Qin Y, et al. Seminal plasma microRNAs: potential biomarkers for spermatogenesis status. Mol Hum Reprod 2012;18:489-97.

[83] Gou LT, Dai P, Liu MF. Small noncoding RNAs and male infertility. Wiley Interdiscipl Rev RNA 2014;5:733-45.

[84] Zhang B, Pan X. RDX induces aberrant expression of microRNAs in mouse brain and liver. Environ Health Perspect 2009; 117:231-40.

[85] Hou L, Wang D, Baccarelli A. Environmental chemicals and microRNAs. Mutat Res 2011;714:105-12.

[86] Sakkas D, Seli E, Manicardi GC, et al. The presence of abnormal spermatozoa in the ejaculate: did apoptosis fail? Hum Fertil (Cambridge) 2004;7:99-103.

[87] Lee E, Ahn MY, Kim HJ, et al. Effect of di(n-butyl) phthalate on testicular oxidative damage and antioxidant enzymes in hyperthyroid rats. Environ Toxicol Pharmacol 2007;22:245-55.

[88] Acharya UR, Rathore RM, Mishra M. Role of vitamin C on lead acetate induced spermatogenesis in swiss mice. Environ Toxicol Pharmacol 2003;13:9-14.

[89] Soubry A, Hoyo C, Jirtle RL, et al. A paternal environmental legacy: evidence for epigenetic inheritance through the male germ line. Bioessays 2014;36:359-71.

[90] Tremellen K. Antioxidant therapy for the enhancement of male reproductive health: a critical review of the literature. In: Parekattil SJ, Agarwal A, editors. Male infertility. 1st ed. New York: Springer; 2012. p. 389-99.

[91] Garolla A, Ghezzi M, Cosci I, et al. FSH treatment in infertile males candidate to assisted reproduction improved sperm DNA fragmentation and pregnancy rate. Endocrine 2017;56:416-25.

[92] Ruvolo G, Roccheri MC, Brucculeri AM, et al. Lower sperm DNA fragmentation after r-FSH administration in functional hypogonadotropic hypogonadism. J Assist Reprod Genet 2013;30:497-503.

[93] Sellami A, Chakroun N, Ben Zarrouk S, et al. Assessment of chromatin maturity in human spermatozoa: useful aniline blue assay for routine diagnosis of male infertility. Adv Urol 2013;2013:578631.

[94] Parmegiani L, Cognigni GE, Filicori M. Sperm selection: effect on sperm DNA quality. Adv Exp Med Biol 2014;791: $151-72$.

[95] Aitken RJ, Finnie JM, Muscio L, et al. Potential importance of transition metals in the induction of DNA damage by sperm preparation media. Hum Reprod 2014;29:2136-217.

[96] Stevanato J, Bertolla RP, Barradas V, et al. Semen processing by density gradient centrifugation does not improve sperm apoptotic deoxyribonucleic acid fragmentation rates. Fertil Steril 2008;90:889-90.

[97] Muratori M, Tarozzi N, Cambi M, et al. Variation of DNA fragmentation levels during density gradient sperm selection for assisted reproduction techniques: a possible new male predictive parameter of pregnancy? Medicine (Baltimore) 2016;95:e3624.

*[98] McDowell S, Kroon B, Ford E, et al. Advanced sperm selection techniques for assisted reproduction. Cochrane Database Syst Rev 2014;10, CD010461.

[99] Gianaroli L, Magli C, Cooldel G, et al. Sperm head's birefringence: a new criterion for sperm selection. Fertil Steril 2008;90:104-12.

[100] Teixeira DM, Barbosa MAP, Ferriani RA, et al. Regular (ICSI) versus ultra-high magnification (IMSI) sperm selection for assisted reproduction. Cochrane Database Syst Rev 2013;7, CD010167. 